\title{
GeoSQL Journey - A gamified learning experience to introduce (or demystify) geospatial SQL queries
}

\author{
Romain Sandoz ${ }^{1}$, Sarah Composto ${ }^{2}$, Sandrine Divorne ${ }^{1}$, Olivier Ertz ${ }^{1}$, \\ and Jens Ingensand ${ }^{2}$
}

Corresponding author:

Olivier Ertz ${ }^{1}$

Email address: olivier.ertz@heig-vd.ch

\section{ABSTRACT}

In a digital world in the making, digital natives develop new learning profiles, interests, and way of working. Simultaneously teachers are facing students with lack of engagement and motivation with quite traditional learning process that has probably to be reframed considering the effects of digital transformation in the education sector. This issue is acute when it comes to complex subject of study, such as SQL geospatial to manipulate the geospatial characteristic of data. Indeed, some common difficulties have been identified by teachers from HEIG-VD university both in Media Engineering and Geomatics fields of study. The user-centered approach aims at creating digital products highly responding to the user's needs through techniques improving the user experience. Various aspects have to be considered, including emotions. In education, gamification, along with user experience, interface design and usability best practices is one promising approach able to increase the learner's engagement, interest and motivation. It aims to implement game mechanics within non-game context, in order to motivate the learner to accomplish a task and increase the ability to learn new skills. Using a gamification layer within a given context, being digital or not, act as a motivational trigger. It helps giving meaningful, enjoyable and empowering experience. SQL Island is a project from Kaiserslautern University of Technology which illustrates very well a gamified learning experience of the SQL special-purpose programming language. The GeoSQL Journey project goes further, tackling SQL geospatial to learn in a fun way how to manipulate the geospatial characteristic of data. It is a gamified pedagogical application to introduce the students to the practice of SQL geospatial during the first hours or days of the course. Serving as an initiation, it is designed to focus on intrinsic motivation (personal development, quest, challenge and fulfillment) with learning objectives determined and integrated with an engaging and coherent game world and narrative. This paper describes the early work of conceptual design of the GeoSQL Journey project. Game mechanics and game interface has been conceived and brought together according to the literature in the domain and best practices on this matter. The following step for this project is to elaborate a testing method without yet having to develop an application prototype (e.g. organizing a fairly raw tabletop game associated with a classic SQL console) so as to challenge the design with students and teachers to get their feedbacks. Also, it is envisioned to evaluate how existing open source gamification tools and frameworks would be suitable to develop the first prototype planned for the 2019-2020 academic year.

\section{INTRODUCTION}

As digital natives, today students fully experience the digital transformation and develop new learning profiles, needs, preferences and requirements. While teachers are facing new challenges to engage and motivate them to participate in the learning process, the digital transformation also offers opportunities to build new "techno-pedagogical" approaches. For instance, given the trend that coding is a new literacy (Vee, 2017), interesting ideas and interactive environments have been developed for learners not 
necessarily computer science professionals (Code.org, Code Academy, SqlZoo, Galaxql). Some of these are using gamification principles which can be used for various purposes, especially for education.

SQL Island (Schildgen, 2014) is a project which illustrates very well a gamified learning experience of the SQL special-purpose programming language. The GeoSQL Journey project goes further, tackling SQL geospatial (GeoSQL hereunder) to learn in a fun way how to manipulate the geospatial characteristic of data. The intent is to provide teachers and learners a useful tool as an introduction course about GeoSQL that helps demystify the first impression of complexity that emerges from geospatial queries and to rather reveal its power in a fun way.

The motivation for such a project finds its roots in the difficulties encountered by teachers from HEIG-VD university both in Media Engineering and Geomatics fields of study. And finally the idea came up to consider Gabe Zichermann's point (Zichermann and Cunningham, 2011) that game technics could increase by $40 \%$ the ability to learn new skills.

\section{GAMIFICATION IN THEORY}

Implemented in the learning process, gamification aims to increase students' engagement and curiosity, also to activate incentives specific to each individual in order to accomplish a task. The gamification process 1 does enhance $\ll$ services with (motivational) affordances in order to invoke gameful experiences and further behavioral outcomes $\gg$ (Hamari et al., 2014) while reducing the negative emotions coming from traditional learning process.

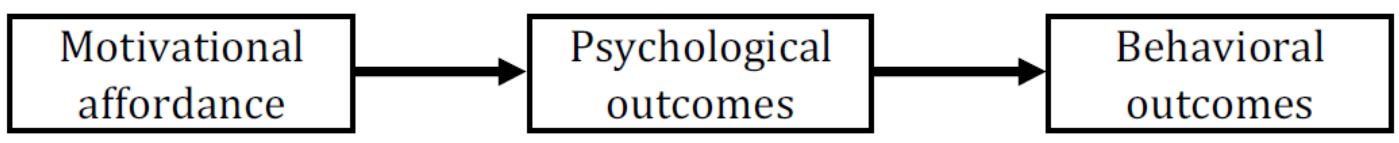

Figure 1. Definition of gamification process.

Even a parallel can be made between education and game mechanics. Indeed, students are training to learn specific skills, pass tests, get $\ll$ rewards $\gg$ (grades) and increase their $\ll$ level $\gg$ at the start of each new academic year. However gamified mechanics aim less practical but important motivational components such as creativity and emotions (Lee and Hammer, 2011).

Furthermore, gamification allows four freedoms: to experiment, to fail, to explore multiple identities, to control one's own investment and experience (Lee and Hammer, 2011). For instance, failure is considered as an option. In video games it could be for an entire level where you fail and fail again until you succeed, even for very small tasks. Yet, the same person might have a strong negative feeling and discouragement when getting a bad or not so perfect grade after a school exam, precisely because failure is not an option, not accepted (Schwartz, 2017).

Since GeoSQL Journey is an initiation to geospatial SQL with a rather short lifespan and no long-term retention of the learner, it is designed to focus on intrinsic motivation rather than extrinsic motivation. Therefore, personal development, quest, challenge and fulfillment are favoured over points, badges and leaderboard. Moreover, the different learning objectives are determined and integrated in an engaging narration. Just as in SQL Island, the user must carry out missions by speaking SQL (the only language spoken by inhabitants of an island) but with the geospatial dimension added to the language and embedded in the game world.

\section{GAMIFICATION IN ACTION}

The current focus of the project is to design the gamified learning experience: from learning process in relation to pedagogical aspects, game mechanics, difficulty levels and game design to visual design. As an actual result, a first user interface has been designed considering gamification best practices in relation to user experience design. Along with it, the game world has been established. Then the game mechanics has been built according to the pedagogical objectives, including a tutorial and a first game level divided in learning steps. Since the knowledge of the basics of SQL is a prerequisite, the tutorial aims to present not only the game interface 2, but also to bring the first elements of understanding of GeoSQL. Then, the first game level goes further, introducing and training step-by-step GeoSQL instructions according to a learning process 3 . 


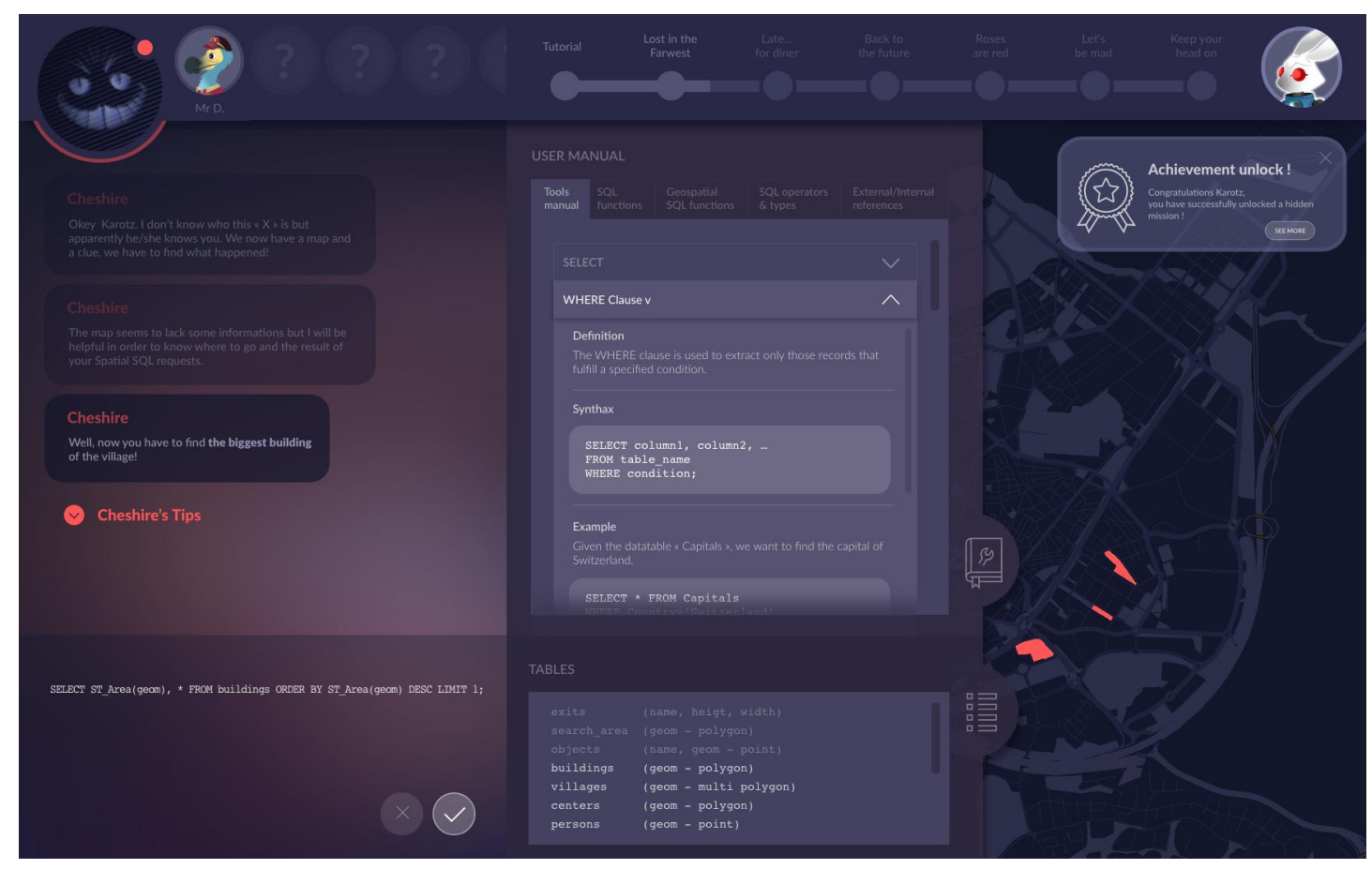

Figure 2. Game interface (top: progress bar; left: SQL console and dialog window telling the story; middle: documentation and inventory of game objects to interact with (table description); right: map of the game (in red, selected objects resulting from a GeoSQL query).

The game interface is composed of several features. The main element is an SQL editor, which is the central interaction tool to progress in the game. As motivational feedback, visuals and rewards appear if the user enters the correct query statement. Feedbacks are given not only for correct answers. Bad answers also give visual or textual feedback to the user in order to minimize the impact of failure and enhance the freedom to fail. Moreover, the game interface contains a map in order to represent the geospatial dimension. If the user enters the correct query statement, the result is shown on the map. For instance, at the beginning of the first level, users must find the biggest building of the village. If the user enters the correct query statement, the map jumps to a tighter, zoomed-in view of this building. The progress bar is another visual element of the interface. It helps the user follow the progress made in the game and to know what has to be done.

\section{FROM PEDAGOGICAL OBJECTIVES TO GAME WORLD}

Designing the mechanics that link the game world with the pedagogical objectives is a difficulty in itself. As an example, in our design, this can be illustrated by the $\ll$ scanner $\gg$ game tool. Considering pedagogical objectives related to the use of ST_Buffer and ST_Within instructions, this tool does combine both instructions to enable an action in the game world (herein scan it around the user to find a person or a key to open a door):

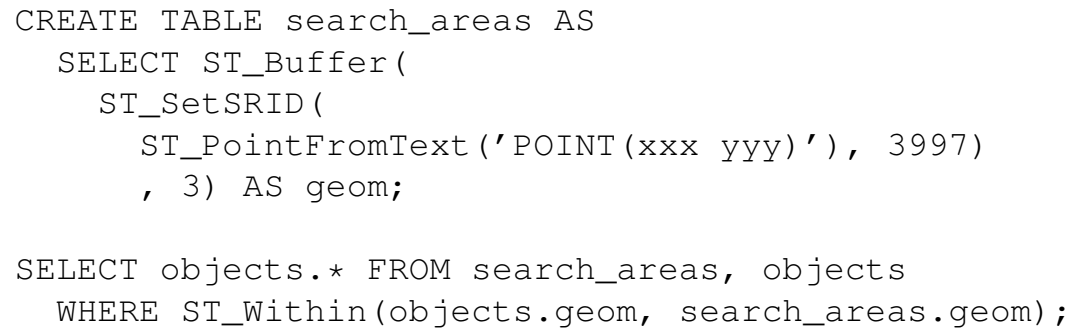

When you learn something new and rather difficult like GeoSQL, you may need special support. To gamify this idea of support, different kinds of helper are provided throughout the game. Firstly, the user's 


\begin{tabular}{|l|c|c|c|c|c|c|}
\hline \multicolumn{1}{|c|}{ Spatial method } & \multirow{2}{*}{ Tutorial } & \multicolumn{5}{|c|}{ Level 1 } \\
\hline ST_Area & Step 1 & Step 2 & Step 3 & Step 4 & Step 5 \\
\hline ST_Buffer & $\mathrm{x}$ & $\mathrm{x}$ & & & & \\
\hline ST_Centroid & & & $\mathrm{x}$ & $\mathrm{x}$ & & \\
\hline ST_Contains & & & $\mathrm{x}$ & & & \\
\hline ST_Difference & & & & $\mathrm{x}$ & & \\
\hline ST_Intersection & & & & & $\mathrm{x}$ & \\
\hline ST_PointFromText & $\mathrm{x}$ & & & & & \\
\hline ST_SetSRID & $\mathrm{x}$ & & & $\mathrm{x}$ & & \\
\hline ST_Transform & & & & & & $\mathrm{x}$ \\
\hline ST_Union & & & $\mathrm{x}$ & & & \\
\hline ST_Within & $\mathrm{x}$ & & & $\mathrm{x}$ & & \\
\hline ST_X & & & & & & $\mathrm{x}$ \\
\hline ST_Y & & & & & & $\mathrm{x}$ \\
\hline
\end{tabular}

Figure 3. Geospatial learning process of GeoSQL Journey.

character - the hero of the game - is assisted by a companion, which will guide the learner during the quest, providing tips to help to build the desired SQL query statement. For instance, when the user enters a very first query statement, the companion reminds us how to build a SQL query statement: SELECT [field(s)] FROM [table]. In a second step, the companion may suggest to use the keyword WHERE to refine the result and produce the right action in the game to progress.

Beside the companion, the user has a documentation which contains definitions, syntactic rules and examples of instructions supposed to be known and used all along the game. In the proposed game design, this documentation is completed step-by-step, almost empty at the beginning, it grows as the user goes further. We may even think to the game technique by introducing a virtual currency allowing the user to buy tips and tricks to help finding the solution.

Also the progress in term of difficulties is considered with some query statements partially or totally provided. This kind of helper dwindles as the user goes further and thus increases the difficulty and get the user to find answers by himself. During the tutorial, almost all query statements are totally provided, whereas users must partially enter almost all query statements at the middle of game and totally at the end of the game, reusing query statements learned previously and the documentation.

Finally, given that some nested and cross-joined queries are often difficult to grasp at the early stages of learning, the game design proposes "facilitation tools" so as to avoid negative feeling and still favour assisted learning. For example, the "auto-save" tool which does automatically save intermediate query results in a temporary storage (e.g. creating a table). Later in the game, the tool may go « out of service $\gg$ constraining the user to solve the problem without it, as explained in the documentation and may be still with a bit help from the companion.

\section{CONCLUSION AND PERSPECTIVES}

After this first iteration of game design, the next phase would be to implement a web-based prototype. But a preliminary step is envisioned, that is, to test elements of the design in classroom with some «low-fidelity » material to simulate the playing of the game. The exact testing methodology has to be defined without having to develop specific pieces of software and may be reusing existing pieces when relevant.

There are many web technologies useful to develop a solution from scratch, but also some serious game frameworks like Wegas authoring system (Jaccard, 2018), the Tourney content independent game 
framework (Dornberger et al., 2014) or even Makahiki, a serious game framework for sustainability (Xu et al., 2014). Nonetheless, the usefulness of the many existing gamification tools, both closed and open source, has to be evaluated, especially when the game design is not just about classical quizzes, points, badges and leaderboard, but rather built of a specific narration and creative game world.

Also, beside the fact that we favor open source solutions especially for projects supported by public funds, we think that open source solutions should be favored to develop open education software. Not only because of the shared word $\ll$ open $\gg$ but above all to streamline collaborations between universities.

Finally, GeoSQL Journey will be tested with students and teachers to get their feedbacks. Since it is a first design iteration, many pedagogical aspects in relation to game techniques have still to be improved and newly considered.

\section{ACKNOWLEDGMENTS}

We would like to thank the Geographic Information Technology Training Alliance (gitta.info) for funding this exploratory project in the frame of its Content Development Initiative (CDI).

\section{REFERENCES}

Dornberger, R., Korkut, S., Hil, D., and Jäger, J. (2014). Tourney-How to Gamify Learning with Design and Technology. European Conference on Games Based Learning, 2:812.

Hamari, J., Koivisto, J., and Sarsa, H. (2014). Does Gamification Work? - A Literature Review of Empirical Studies on Gamification. In 2014 47th Hawaii International Conference on System Sciences, pages 3025-3034, Waikoloa, HI. IEEE.

Jaccard, D. (2018). A Web Game Authoring System. original-date: 2012-01-04T13:31:04Z.

Lee, J. J. and Hammer, J. (2011). Gamification in Education: What, How, Why Bother?

Schildgen, J. (2014). SQL Island: An Adventure Game to Learn the Database Language SQL.

Schwartz, M. (2017). Teaching with Gamification.

Vee, A. (2017). Coding Literacy: How Computer Programming Is Changing Writing. MIT Press, Cambridge, MA.

Xu, Y., Johnson, P. M., Lee, G. E., Moore, C. A., and Brewer, R. S. (2014). Makahiki: An Open Source Serious Game Framework for Sustainability Education and Conservation.

Zichermann, G. and Cunningham, C. (2011). Gamification by design: implementing game mechanics in web and mobile apps. O’Reilly Media, Sebastopol, Calif, 1st. ed edition. OCLC: ocn711045699. 